Check for updates

Cite this: RSC Adv., 2017, 7, 26371

Received 30th March 2017

Accepted 30th April 2017

DOI: $10.1039 / \mathrm{c} 7 \mathrm{ra03672h}$

rsc.li/rsc-advances

\title{
A visible high efficiency and polarization- insensitive 34-level dielectric metasurface hologram
}

\author{
Xiaofang Su, (D) ab Guanhai Li, ${ }^{\text {*a }}$ Hui Yang, ${ }^{\text {ab }}$ Zengyue Zhao, ${ }^{\text {ac }}$ Feilong Yu, ${ }^{\text {ab }}$ \\ Xiaoshuang Chen*a and Wei Lu ${ }^{a}$
}

\begin{abstract}
We propose a dielectric metasurface of 34 phase level, to reproduce the holographic image in the visible region. With normal incident circular polarized illumination, imaging efficiency as high as $59.72 \%$ is achieved at $560 \mathrm{~nm}$ wavelength. Consisting of non-resonant meta-cells, the proposed polarizationinsensitive dielectric metasurface performs well in a broad band from $480 \mathrm{~nm}$ to $660 \mathrm{~nm}$. Numerical results also indicate that the metasurface hologram maintains high fidelity within a wide-angle incidence $\left(0-25^{\circ}\right)$, and show high tolerance to fabrication errors. The high efficiency, broadband, wide-angle, and high fabrication tolerance metasurface based on the hologram, can be used in the fields of display, security, data storage, and information processing.
\end{abstract}

\section{Introduction}

Metamaterials, constructed from function-driven artificial metacells, show great potential in tuning electromagnetic properties, such as the effective electric permittivity and magnetic permeability. Several exotic optical phenomena that are barely found in nature $^{1-9}$ are also realized with the proposed design. Metasurfaces, two-dimensional metamaterials composed of quasiperiodic arrays of sub-wavelength structures, retain the abilities to manipulate the amplitude, phase and polarization of light upon transmission or reflection. With the superior control over the propagation of electromagnetic waves, metasurface-based vortex generation, ${ }^{\mathbf{1 0}-13}$ meta-lens, ${ }^{\mathbf{1 3 - 1 9}}$ and wave plates ${ }^{20-22}$ have been demonstrated recently. Compared with bulk 3D metamaterials, the versatile metasurfaces have smaller physical footprint, simpler fabrication and lower losses. These properties endow the metasurface the capability to move traditional bulky optical elements to deep subwavelength scale and pave the pathway for the realization of opto-electronic integrated devices.

Owing to the excellent performance of manipulating electromagnetic waves, metasurface-based holography has been proposed to overcome the defects existing in traditional computer-generated holograms (CGH), for example, low diffraction efficiency caused by unavoidable twin-image and high cost

${ }^{a}$ National Laboratory for Infrared Physics, Shanghai Institute of Technical Physics, Chinese Academy of Sciences, 500 Yu Tian Road, Shanghai, 200083, China. E-mail: ghli0120@mail.sitp.ac.cn; xschen@mail.sitp.ac.cn

${ }^{b}$ University of Chinese Academy of Sciences, No. 19A Yu Quan Road, Beijing, 100049, China

'Shanghai University of Science and Technology, 100 Hai Ke Road, Shanghai, 201210, China in fabrication procedures because of complicated grayscale lithography. In addition, the working wavelength range is wide due to metasurface's flexibility to engineer the electromagnetic properties. Because of these advantages, metasurface-based holography has attracted continuous scientific interest in recent years. ${ }^{18,23-30} \mathrm{Ni}$ et al. ${ }^{26}$ demonstrated a $30 \mathrm{~nm}$ thick plasmonic metasurface hologram in the visible range, which directly provided both the amplitude and phase modulation and generated low-noise images with efficiency of $10 \%$. Zheng et al. ${ }^{27}$ reported a 16-level-phase computer-generated hologram based on a geometric metasurface that reaches polarization conversion efficiency as high as $80 \%$ at $825 \mathrm{~nm}$ and shows a broadband response in near-infrared range.

However, the computer-generated holograms that are realized with metasurface are either limited by polarization dependence or restricted by low efficiency, as reported in ref. 28-30. In our polarization insensitive hologram, near $60 \%$ imaging efficiency is achieved at $560 \mathrm{~nm}$. Built up of $\mathrm{TiO}_{2}$ nanodisks, full $2 \pi$ phase control as well as high transmittance amplitudes are achieved by changing the radius of the nanodisks. Furthermore, we constructed the polarization-insensitive metasurface hologram with 34 phase level meta-cells. In addition to the imaging efficiency, the broadband, wide-angle and high fabrication tolerance characteristics were also investigated to characterize the performance of the polarization-insensitive metasurface hologram.

\section{Model and simulation method}

The designed metasurface composed of $\mathrm{TiO}_{2}$ nanodisks is schematically depicted in Fig. 1. Arranged on fused silica substrate $\left(n_{\mathrm{SiO}_{2}}=1.46\right)$ with fixed period $P=245 \mathrm{~nm}$, the 
nanodisks $\left(n_{\mathrm{TiO}_{2}}=2.4\right)$ have a unified height $H=650 \mathrm{~nm}$. Commercial software based on finite-difference time-domain (FDTD) method is employed to simulate the phase and amplitude of the transmitted light. For the simulation of individual meta-cell's phase shift, periodic boundary conditions are applied at the $x$ and $y$ boundaries and perfectly matched layers (PML) at the $z$ boundaries.

To effectively manipulate the electromagnetic waves, simultaneous full $2 \pi$ phase coverage and high transmittance are necessary. In our model, the phase modulation is achieved by means of the waveguiding effect caused by dielectric metasurface. Each nanodisk is considered as a waveguide and introduces a polarization-insensitive phase shift on the transmitted light. As shown in Fig. 1(d) and (e), with normally incident circular polarized illumination, the transmittance and corresponding phase as a function of radius and period of $\mathrm{TiO}_{2}$ nanodisks are clearly illustrated. Clearly, the phase of the light transmitted from the $\mathrm{TiO}_{2}$ nanodisk metasurfaces can span the entire $2 \pi$ range. Without any significant resonance, the transmission amplitudes show almost uniform high values. This provides the high tolerance of metasurface hologram to fabrication error. For conventional electron beam lithography or reactive ion etching, all the meta-cells' sizes are increased or decreased with the same value for the fabrication error, which have no influence on the phase gradient. To design a high efficiency metasurface hologram, a number of meta-cells with fixed period of $245 \mathrm{~nm}$ are calculated, as shown in Fig. 1(c). When radius varies from $40 \mathrm{~nm}$ to $110 \mathrm{~nm}$ at a $2 \mathrm{~nm}$ interval, $2 \pi$ phase range is achieved. With a pixel dimension of $245 \mathrm{~nm}$, the metasurface hologram is sampled at less than half a wavelength, satisfying the Shannon-Nyquist sampling theorem.
To reproduce our institute's logo 'SITP', a 34-phase level computer-generated metasurface hologram was encoded to have it interfere with the normally incident circular polarized light at a wavelength of $560 \mathrm{~nm}$. The working mechanism of our metasurface is shown in Fig. 2. According to the classical GerchbergSaxton algorithm, a 256 bit grayscale phase distribution of computer-generated hologram was obtained, as shown in Fig. 2(c). In order to effectively simplify the 256 bit hologram, we established an accurate relationship between the radius of the $\mathrm{TiO}_{2}$ nanodisks and phase shift, $r=44 \times \varphi^{3}-93 \times \varphi^{2}+117 \times \varphi$ +41 , with data fitting in MATLAB. This equation provides a straightforward method to convert the phase configuration to radius arrangement. Finally, we chose the 34-phase level to reconstruct the metasurface based hologram. Due to the limitation of computer memory for simulations, the metasurface only consists of $200 \times 200$ evenly spaced $\mathrm{TiO}_{2}$ nanodisks. Radii varying from $42 \mathrm{~nm}$ to $108 \mathrm{~nm}$ at a $2 \mathrm{~nm}$ interval provide a precise phase control to ensure the high fidelity of far field imaging. Compared with conventional multistep lithography processes to fabricate 34-level phase hologram, our holographic metasurface only needs a single lithography step for the same.

\section{Results and discussion}

To characterize the performance of the metasurface-based hologram, far field intensity is calculated using the finitedifference time-domain (FDTD) method. Illuminated by normally incident plane wave with circular polarization, the target image is reproduced, as shown in Fig. 3(b) and (e). Even though only $200 \times 200$ meta-cells are adopted in this study, and the size of the metasurface $(49 \mu \mathrm{m} \times 49 \mu \mathrm{m})$ is much smaller than the reported models, ${ }^{27,29}$ the target holographic image "SITP" and (a)

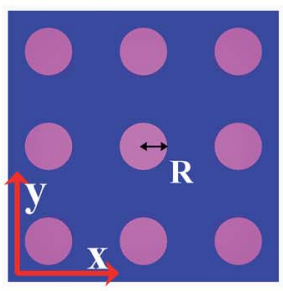

(b)

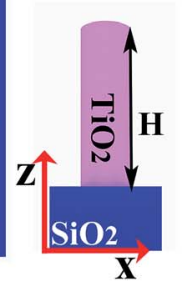

(d)

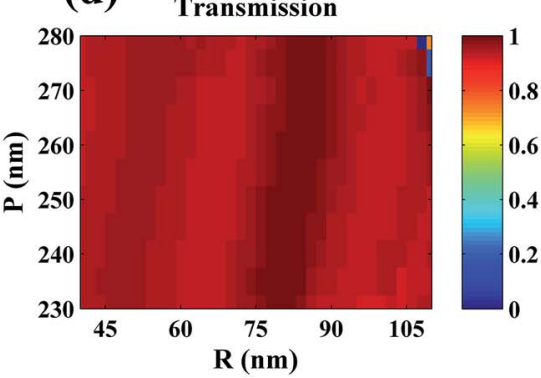

(c)

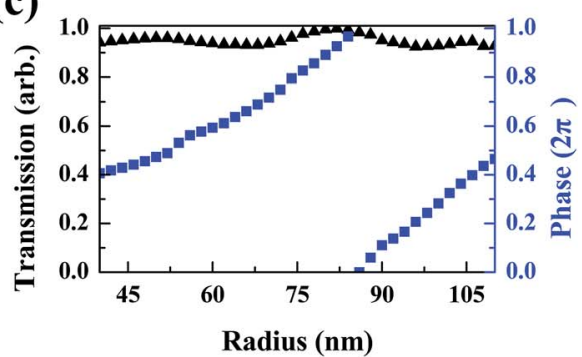

(e)

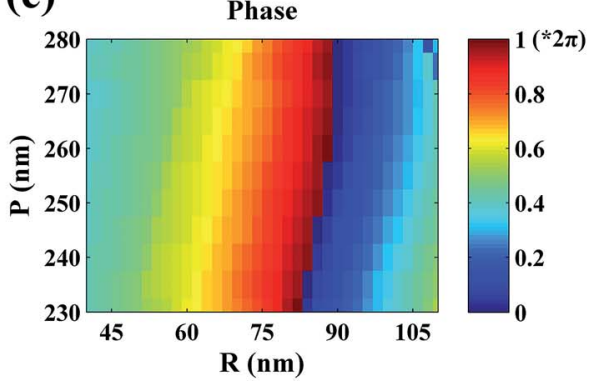

Fig. 1 Schematic structure of metasurface with periodic $\mathrm{TiO}_{2}$ nanodisks $(H=650 \mathrm{~nm}, R=80 \mathrm{~nm}, P=245 \mathrm{~nm}$ ). (a) Top view. (b) Side view. (c) Numerically calculated transmission (black triangle) and phase shift (blue square) for metasurfaces with varied radius and fixed period $P=245 \mathrm{~nm}$ at wavelength $\lambda=560 \mathrm{~nm}$. Transmittance and transmitted light phase variation as a function of $\mathrm{TiO}_{2}$ nanodisk radius $(R)$ and period $(P)$ are shown in (d) and (e), respectively. 
(a)

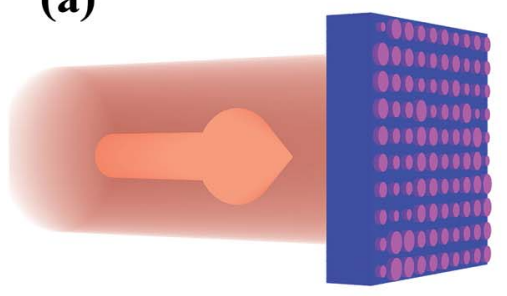

(c)

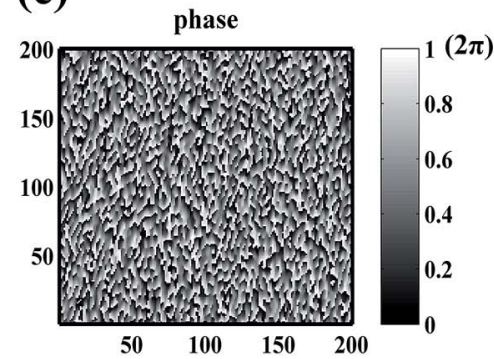

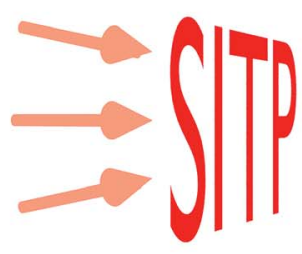

(b)

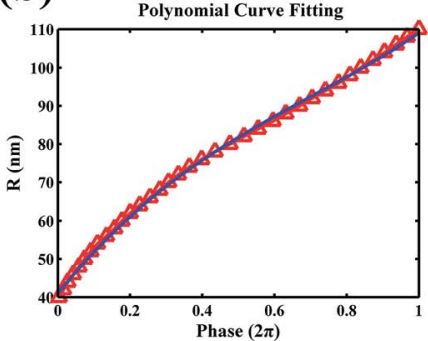

(d)

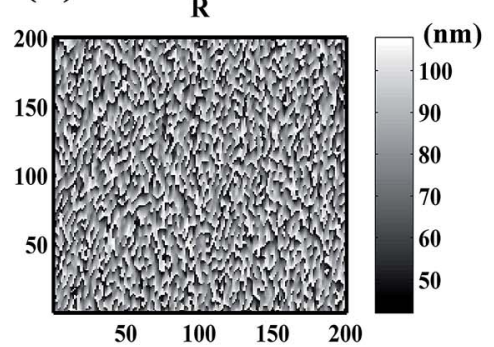

(e)

(f)

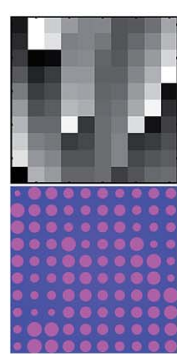

Fig. 2 Design of the metasurface hologram. (a) Conceptual image 'SITP'. (b) Polynomial curve fitting for the transmitted phase shift and radius of the $\mathrm{TiO}_{2}$ nanodisk. The red triangle and blue line represent the simulated result and the corresponding fitting curve, respectively. (c) The computer-generated grayscale phase distribution with $200 \times 200$ pixels designed to reconstruct the target holographic image, and (d) corresponding nanodisk radius distribution $(200 \times 200$ meta-cells). (e) and (f) are the enlarged phase distribution (10 $\times 10$ pixels) and its corresponding meta-cell distribution.

the enlarged " $\mathrm{S}$ " is clearly reproduced. It shows high fidelity with our metasurface hologram. More importantly, no twin image is observed in the simulated far field, which is due to the subwavelength meta-cell's diffraction. Only this one order diffraction property ensures the high imaging efficiency, which is in agreement with our previous result. ${ }^{31}$
To quantitatively evaluate the imaging property of the hologram, we first defined the transmittance efficiency as the ratio of far field intensity going through the metasurface to that passing through the same silica substrate without the $\mathrm{TiO}_{2}$ nanodisk array. Transmission efficiency of $78.15 \%$ was achieved. In addition, we selected the effective pixels from the simulated holographic image by image identification, as shown (a)

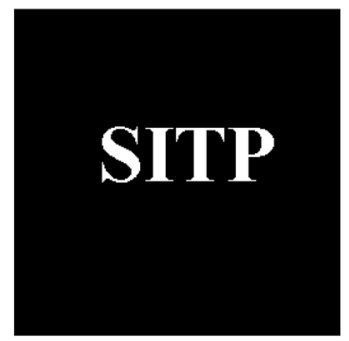

(d)

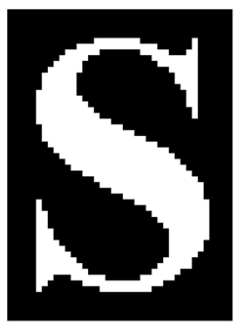

(b)

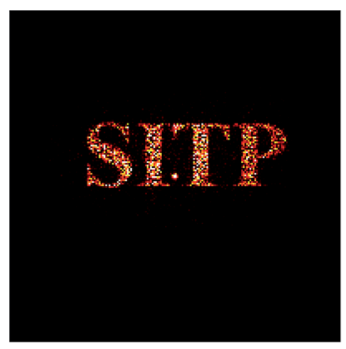

(e)

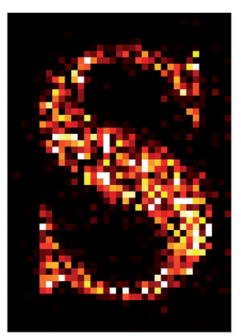

(c)

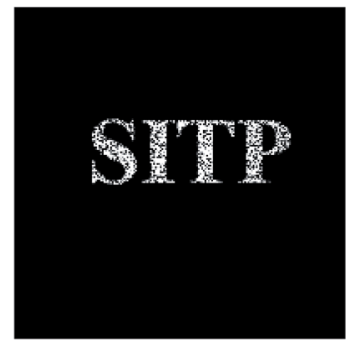

(f)

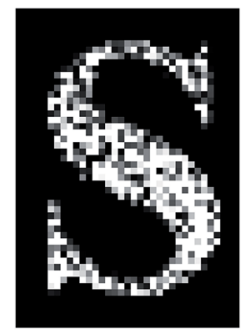

Fig. 3 Image reproduction with a 34-phase level metasurface hologram. (a) The target image 'SITP'. (b) Simulated holographic image acquired by the FDTD method. The far field intensity is normalized. (c) The selected effective pixels used to calculate the imaging efficiency. (d), (e), and (f) show the enlarged letter ' $S$ ' in (a), (b), and (c), respectively. 
in Fig. 3(c) and (f). The conversion efficiency was calculated as the fraction of the total transmitted far field intensity passing through the selected effective area. Herein, $76.41 \%$ of the transmitted far field intensity was converted into the hologram image. Furthermore, we defined the imaging efficiency as the product of transmission efficiency and conversion efficiency. With $59.72 \%$ imaging efficiency of the phase-only hologram, our hologram possesses the highest imaging efficiency on comparison with other polarization-insensitive metasurface based holograms. ${ }^{28-30}$ Moreover, the imaging efficiency can be further enhanced through the addition of more meta-cells or by increasing phase levels.

In addition to the high fidelity and high imaging efficiency, other imaging characteristics such as broadband, wide-angle, and high fabrication tolerance are also investigated to characterize our metasurface hologram. To check the broadband property, far field imaging is calculated for circular polarized incidence at wavelengths of $450 \mathrm{~nm}, 480 \mathrm{~nm}, 514 \mathrm{~nm}$, $553 \mathrm{~nm}, 600 \mathrm{~nm}, 654 \mathrm{~nm}$, as shown in Fig. 4. The intensity distributions clearly illustrate the target holographic image. This demonstrates the excellent broadband property of the metasurface hologram. Essentially, this phenomenon is due to the non-resonant nature of the meta-cells. We also notice that the sizes of the holographic images are enlarged with increasing wavelength. We attribute this to the change of phase accumulation during the propagation, as demonstrated in ref. 25.

The wide-angle imaging property is also demonstrated with FDTD simulations. As shown in Fig. 5, the far field intensities are calculated for oblique incidence at $560 \mathrm{~nm}$ wavelength. With incident angles $\theta_{\mathrm{i}}=5^{\circ}, 10^{\circ}, 15^{\circ}, 20^{\circ}, 25^{\circ}$, the zeroth-order (a) $\lambda=450 \mathrm{~nm}$

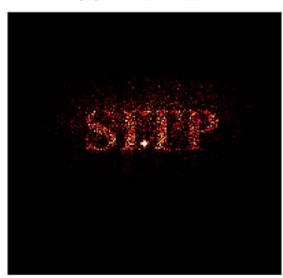

(d) $\lambda=553 \mathrm{~nm}$

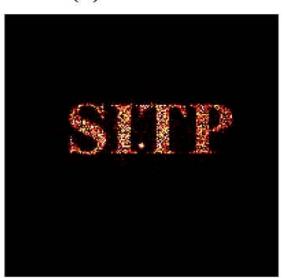

(b) $\lambda=480 \mathrm{~nm}$

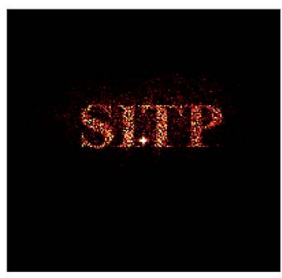

(e) $\lambda=600 \mathrm{~nm}$

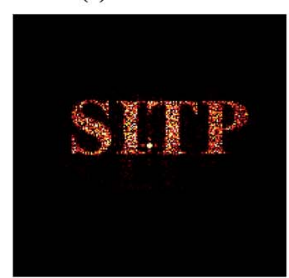

(c) $\lambda=514 \mathrm{~nm}$

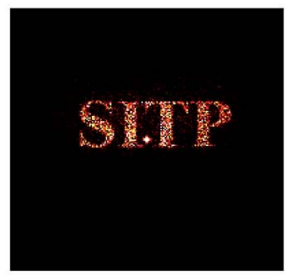

(f) $\lambda=654 \mathrm{~nm}$

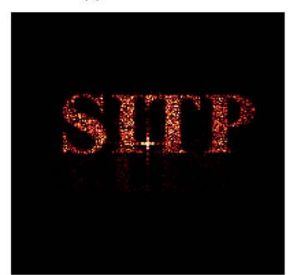

Fig. 4 Broadband property of the hologram. Far field intensity images simulated at the wavelength of (a) $450 \mathrm{~nm}$, (b) $480 \mathrm{~nm}$, (c) $514 \mathrm{~nm}$, (d) $553 \mathrm{~nm}$, (e) $600 \mathrm{~nm}$, (f) $654 \mathrm{~nm}$.

(a) $\theta \mathrm{i}=0^{\circ}$

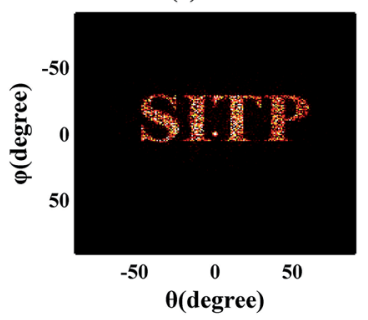

(d) $\theta \mathrm{i}=15^{\circ}$

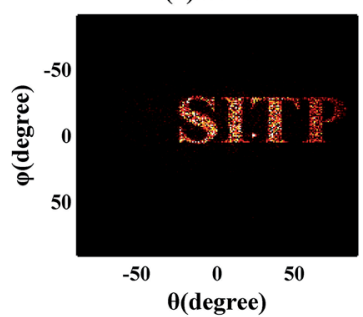

(b) $\theta \mathrm{i}=5^{\circ}$

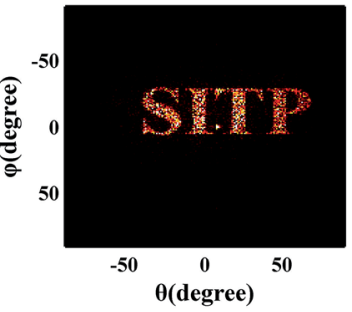

(e) $\boldsymbol{\theta i}=\mathbf{2 0}$

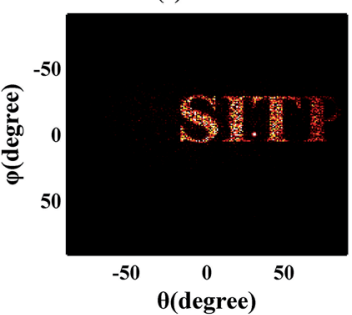

(c) $\theta \mathrm{i}=10^{\circ}$

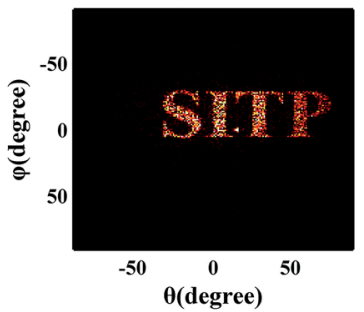

(f) $\theta \mathrm{i}=25^{\circ}$

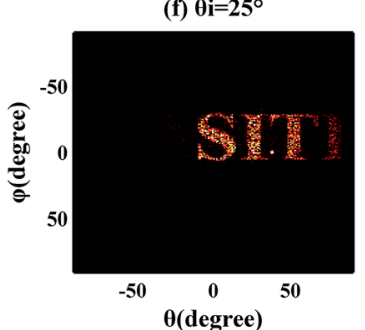

Fig. 5 Imaging dependence on the incidence angle. Far field intensities for the metasurface hologram simulated with incident angle (a) $0^{\circ}$, (b) $5^{\circ}$, (c) $10^{\circ}$, (d) $15^{\circ}$, (e) $20^{\circ}$, (f) $25^{\circ}$, at designed wavelength. 
(a) Tolerance (Ri-5nm)

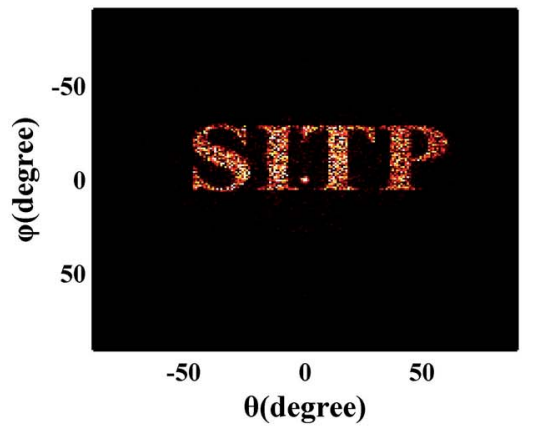

(c) Tolerance $(\mathrm{Ri}+\mathbf{1 0 n m})$

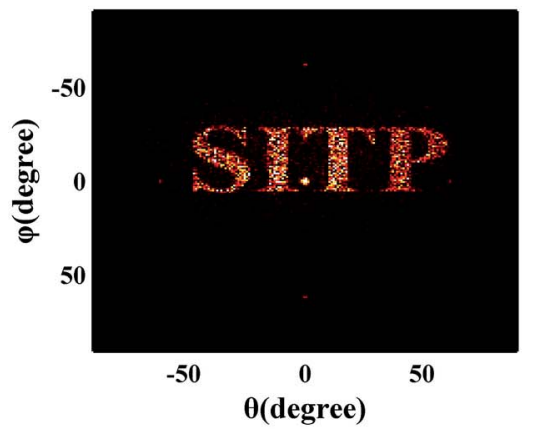

(b) Tolerance (Ri-10nm)

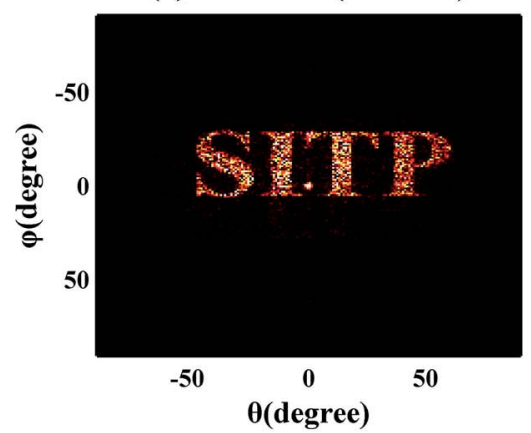

(d) Tolerance (Ri \pm random(1 5nm))

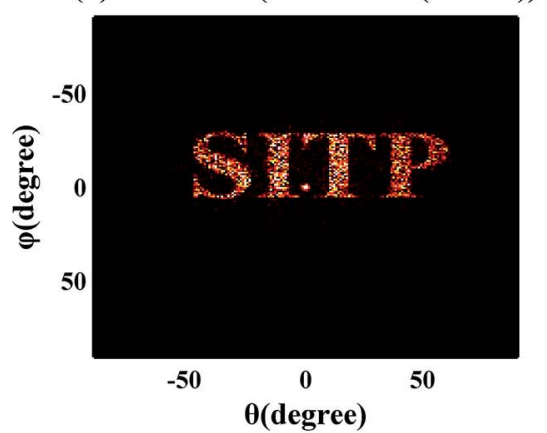

Fig. 6 High tolerance to fabrication errors. Simulated far field intensities for metasurface with different nanodisks. All nanodisks' radii with (a) minus $5 \mathrm{~nm}$, (b) minus $10 \mathrm{~nm}$, (c) plus $10 \mathrm{~nm}$ at designed $R_{\mathrm{j}}$. (d) Every nanodisk adds a random error $( \pm 1-5 \mathrm{~nm}$ ).

beams focus at spots with deviation angles $\theta=8^{\circ}, 15^{\circ}, 23^{\circ}, 31^{\circ}$, $38^{\circ}$ respectively. With $76.41 \%, 72.69 \%, 67.65 \%, 61.93 \%, 55.98 \%$ transmittance efficiency, the target holographic image is reconstructed clearly at the five incident angles except the blurry "P" at $\theta_{\mathrm{i}}=25^{\circ}$. The wide-angle imaging property is because of the selected non-resonance $\mathrm{TiO}_{2}$ nanodisks, which are insensitive to oblique incidence. Herein, we want to emphasize that our metasurface-based hologram not only has no polarization dependence on the reference beam, but also lowers the collimation demand of incidence light.

To promote the application of metasurface in large-scale holographic technology, keeping a large fabrication tolerance is important to ensure the performance of the fabricated device. In this study, uniform and random dimension errors caused by the electron beam lithography or reactive ion etching are both considered, as shown in Fig. 6. With $62 \%$, $61.6 \%, 51.9 \%, 57.23 \%$ imaging efficiency, the holographic images are represented without any significant degradation of the performance, as shown in Fig. 6. In this case, due to the approximate linear relationship of radius and phase shift, the phase gradient can be seen as a constant, which ensures the high imaging efficiency. This promises that our polarizationinsensitive meta-hologram has a very good tolerance to the fabrication error.

\section{Conclusion}

In conclusion, we proposed a metasurface of 34-phase level based hologram in the visible range. With high fidelity, the micrometer-scale metasurface hologram can achieve an imaging efficiency as high as $59.72 \%$ at $560 \mathrm{~nm}$ wavelength. In addition, we also demonstrated the imaging properties with broadband, wide-angle, and high fabrication tolerance for the metasurface hologram. With such excellent performance, the dielectric metasurface can be used in holographic display, high resolution holographic data storage, optical information processing etc.

\section{Acknowledgements}

The authors acknowledge the support provided by the State Key Program for Basic Research of China (2013CB632705, 2011CB922004), the National Natural Science Foundation of China (10990104, 11334008, and 61290301), the Fund of Shanghai Science and Technology Foundation (13JC1408800, 16ZR1445300), Shanghai Sailing Program (16YF1413200), and Youth Innovation Promotion Association CAS (2017285).

\section{References}

1 R. A. Shelby, D. R. Smith and S. Schultz, Science, 2001, 292(5514), 77-79.

2 C. M. Soukoulis, S. Linden and M. Wegener, Science, 2007, 315(5808), 47-49.

3 D. Ye, K. Chang, L. Ran and H. Xin, Nat. Commun., 2014, 5, 5841.

4 S. Xi, H. Chen, T. Jiang, L. Ran, J. Huangfu, B. I. Wu, J. A. Kong and M. Chen, Phys. Rev. Lett., 2009, 103, 194801. 
5 S. N. Galyamin, A. V. Tyukhtin, A. Kanareykin and P. Schoessow, Phys. Rev. Lett., 2009, 103, 194802.

6 P. Genevet, D. Wintz, A. Ambrosio, A. She, R. Blanchard and F. Capasso, Nat. Nanotechnol., 2015, 10(9), 804-809.

7 S. H. Lee, C. M. Park, Y. M. Seo and C. K. Kim, Reversed Doppler effect in double negative metamaterials, Phys. Rev. B: Condens. Matter Mater. Phys., 2010, 81, 241102.

8 D. Ziemkiewicz and S. Zielinska-Raczynska, J. Opt. Soc. Am. B, 2015, 32(3), 363-369.

9 J. Ran, Y. Zhang, X. Chen, K. Fang, J. Zhao, Y. Sun and H. Chen, Sci. Rep., 2015, 5, 11659.

10 N. Yu, P. Genevet, M. A. Kats, F. Aieta, J. P. Tetienne, F. Capasso and Z. Gaburro, Science, 2011, 334(6054), 333-337.

11 L. Huang, X. Chen, H. Mühlenbernd, G. Li, B. Bai, Q. Tan, G. Jin, T. Zentgraf and S. Zhang, Nano Lett., 2012, 12(11), 5750-5755.

12 Y. Yang, W. Wang, P. Moitra, I. I. Kravchenko, D. P. Briggs and J. Valentine, Nano Lett., 2014, 14(3), 1394-1399.

13 X. Ma, M. Pu, X. Li, C. Huang, Y. Wang, W. Pan, B. Zhao, J. Cui, C. Wang, Z. Zhao and X. Luo, Sci. Rep., 2015, 5, 10365.

14 X. Chen, L. Huang, H. Mühlenbernd, G. Li, B. Bai, Q. Tan, G. Jin, C. Qiu, S. Zhang and T. Zentgraf, Nat. Commun., 2012, 3, 1198.

15 F. Aieta, P. Genevet, M. A. Kats, N. Yu, R. Blanchard, Z. Gaburro and F. Capasso, Nano Lett., 2012, 12(9), 4932-4936.

16 X. Ni, S. Ishii, A. V. Kildishev and V. M. Shalaev, Light: Sci. Appl., 2013, 2(4), e72.

17 D. Lin, P. Fan, E. Hasman and M. L. Brongersma, Science, 2014, 345(6194), 298-302.

18 A. Arbabi, Y. Horie, M. Bagheri and A. Faraon, Nat. Nanotechnol., 2015, 10, 937-943.
19 M. Khorasaninejad, A. Y. Zhu, C. Roques-Carmes, W. T. Chen, J. Oh, I. Mishra, R. C. Devlin and F. Capasso, Nano Lett., 2016, 16(11), 7229-7234.

20 N. Yu, F. Aieta, P. Genevet, M. A. Kats, Z. Gaburro and F. Capasso, Nano Lett., 2012, 12(12), 6328-6333.

21 Z. H. Jiang, L. Lin, D. Ma, S. Yun, D. H. Werner, Z. Liu and T. S. Mayer, Sci. Rep., 2014, 4, 7511.

22 F. Ding, Z. Wang, S. He, V. M. Shalaev and A. V. Kildishev, ACS Nano, 2015, 9(4), 4111-4119.

23 B. Wang, F. Dong, Q. T. Li, D. Yang, C. Sun, J. Chen, Z. Song, L. Xu, W. Chu, Y. F. Xiao, Q. Gong and Y. Li, Nano Lett., 2016, 16(8), 5235-5240.

24 E. Almeida, O. Bitton and Y. Prior, Nat. Commun., 2016, 7, 12533.

25 L. Huang, X. Chen, H. Mühlenbernd, H. Zhang, S. Chen, B. Bai, Q. Tan, G. Jin, K. W. Cheah, C. W. Qiu, J. Li, T. Zentgraf and S. Zhang, Nat. Commun., 2013, 4, 2808.

26 X. Ni, A. V. Kildishev and V. M. Shalaev, Nat. Commun., 2013, 4, 2807.

27 G. Zheng, H. Mühlenbernd, M. Kenney, G. Li, T. Zentgraf and S. Zhang, Nat. Nanotechnol., 2015, 10(4), 308-312.

28 Q. T. Li, F. Dong, B. Wang, F. Gan, J. Chen, Z. Song, L. Xu, W. Chu, Y. F. Xiao, Q. Gong and Y. Li, Opt. Express, 2016, 24(15), 16309-16319.

29 K. E. Chong, L. Wang, I. Staude, A. R. James, J. Dominguez, S. Liu, G. S. Subramania, M. Decker, D. N. Neshev, I. Brener and Y. S. Kivshar, ACS Photonics, 2016, 3(4), 514-519.

30 W. Zhao, H. Jiang, B. Liu, J. Song, Y. Jiang, C. Tang and J. Li, Sci. Rep., 2016, 6, 30613.

31 G. Li, B. P. Clarke, J. K. So, K. F. MacDonald and N. I. Zheludev, Nat. Commun., 2016, 7, 13705. 